\title{
A Novel Approach for Identification and Ranking of Road Traffic Accident Hotspots
}

\author{
El-Said M. M. Zahran , Soon Jiann Tan, Yok Hoe Yap, Ena K. A. Rahman and Nurulhikmah H. Husaini \\ Centre for Transport Research, Universiti Teknologi Brunei, Brunei Darussalam
}

\begin{abstract}
Road Traffic Accidents (RT A) are known to be one of the main causes of fatalities worldwide. One useful approach to improve road safety is through the identification of RT A hotspots along a road, so they can be prioritised and treated. This paper introduces an approach based on Geographical Information System (GIS) to identify and prioritise RT A hotspots along a road network using historical RT A data. One particular urban road in Brunei with a historically high rate of RT As, Jalan Gadong, was selected as a case study. Five years of historical RT A data were acquired from the relevant authorities and input into a GIS database. GIS analysis was then used to identify the spatial extension of the RTA hotspots. The RT A hotspots were ranked according to three different schemes: frequency, severity and socio-economic impact of RT As. A composite ranking scheme was also developed to combine these schemes; this enabled the prioritisation and development of intervention and maintenance programmes of the identified RT A hotspots. A visualisation method of the RT A spatial distribution within each identified RT A hotspot was also developed to determine the most risky road stretches within each hotspot, which is important for treatment prioritisation when limited resources are available.
\end{abstract}

\section{Introduction}

In 2013, the number of human injuries and fatalities resulting from Road Traffic Accidents (RTAs) alone was estimated to be 1.25 million worldwide [1, 2]. These RTAs have adverse impacts, directly and indirectly, on both national economics as well as health consequences and are seen to be the primary cause of fatalities among those aged between 15 and 29 in 2012 [2]. Developing countries that are experiencing rapid urban sprawl and rise in motorised traffic are those who suffers the escalating number of RTAs the most [3]. Comparatively slow pace of development in road infrastructure, levels of enforcement and policies became one of the main factors that are contributing to the rapid increase of the RTAs [4].

Brunei Darussalam is a small country that is located along the Northern coastline of Borneo Island on the Southern region of the South China Sea with a total land area of 5,770 square kilometres. It has a tropical climate and high humidity temperature and heavy rainfall uniformly throughout the year. In 2014, it was recorded that Brunei has a total road network of $3,191.2 \mathrm{~km}$ and a total population of 411,900 with $1.4 \%$ annual growth rate at which $69.6 \%$ of the entire population are concentrated within the Brunei-Muara District [5]. Community within Brunei depend heavily on cars rather than other transportation at which $92 \%$ of the traffic fleet are private cars. With this percentage, Brunei has the highest vehicles to population ratio in the world [6]. Predominance of private cars in Brunei is mainly due to the inadequate availability of public transport, dispersal of urban developments and even low price of car and fuel [7]. W ith the high number of private cars, RTA rate is higher than various developed and developing countries which results in significant social and economic impacts on the country [8].

One tool that can be used effectively to achieve a sustainable transportation planning and sustainable traffic management in urban areas is Geographic Information System (GIS) [4]. GIS also provide powerful tools in developing reliable geospatial databases that can be used when analysing RTA data [9].

In India, GIS was used to identify RTA hotspots along a road section that is known for accidents [10]. With the help of GIS, they visually identified locations along the road section that had a high rate of RTA. A crash factor, based on RTA rate, was computed for each of these locations. The crash factors were then compared to an empirical critical crash rate that was computed for the entire study road. If the crash rate is seen to exceed the critical crash rate, location is classified as an accident hotspot. However, their approach did not include the use of GIS analysis nor did they determine the spatial extent or even to rank the identified accident hotspots.

For a national highway in Algeria, Ref. [11] conducted hotspot analysis in GIS using Kernal Density Estimation (KDE) method to identify and priorit ise those RTA hotspots. KDE method was used to trans form RTA data, in discrete point form, into a continuous raster coverage that displayed the density distribution of RTA 
along the selected highway. The acquired coverage applied an arbitrary search with a radius of $0.5 \mathrm{~km}$, and the output cell size was not specified. No proper justification was made as to why $0.5 \mathrm{~km}$ search radius was selected even though it has a significant impact on the prediction accuracy of the kernel density estimation method in GIS $[12,13]$. Aside from that, this method can only be used to rank accident hotspots based on the frequency of RTA.

The following sections of this paper demonstrate an approach using GIS to identify and delineate the spatial extension of RTA hotspots along a case-study road in Brunei Darussalam. The GIS approach also priorit ises the identified hotspots using four different ranking schemes that are based on frequency, risk factor, economic impact of RTA within each hotspot zone and the combination of these three criteria.

\section{Digitisation and geocoding of past RTA data}

As a result of preliminary analysis of RTA cases, major accident-prone roads in Brunei Darussalam were identified. Jalan Gadong was selected as a case study for this research paper due its high number of reported accidents per $\mathrm{km}$ per year.

Jalan Gadong is a $10.2 \mathrm{~km}$ long dual carriageway connecting Jalan Kumbang Pasang in the east and Jalan Tutong to the west of the capital city of Brunei, Bandar Seri Begawan. As a major urban arterial carrying traffic from the west of the capital while providing access to various commercial buildings, residential houses and schools along its length, it has a relatively very high usage rate, estimated to be over 47,000 vehicles per day [14]. There are 9 signalised junctions, 1 signalised roundabout, 15 mid-block U-turns and many more uncontrolled junctions along the whole stretch of road. The posted speed limit is $65 \mathrm{~km} / \mathrm{hr}$ throughout, except for an approximately $1 \mathrm{~km}$ long section with a $50 \mathrm{~km} / \mathrm{hr}$ speed limit due to the presence of several uncontrolled junctions.

During the initial stage of data digitisation, RTA data for Jalan Gadong was exported manually from standard police report form into a spreadsheet program. The data was further filtered, cleaned and refined to ensure only reports with sufficient location details were used for GIS analysis. Suitable data field and coding systems were also developed to indicate the crash movement type and contributory factors of each case. As for the coordinates of the RTA location, they were estimated using both digital maps and handheld GPS units.

In all, RTA data of Jalan Gadong within the period of 2011 to 2015 were processed, comprising of 747 accident cases with 4 fatalities, 18 serious injuries, 52 slight injuries and 1262 no injuries for both traffic directions of the dual carriageway, as shown in Figure 1. The most common type of crashes was rear-end crashes, and involved 2 or more vehicles.

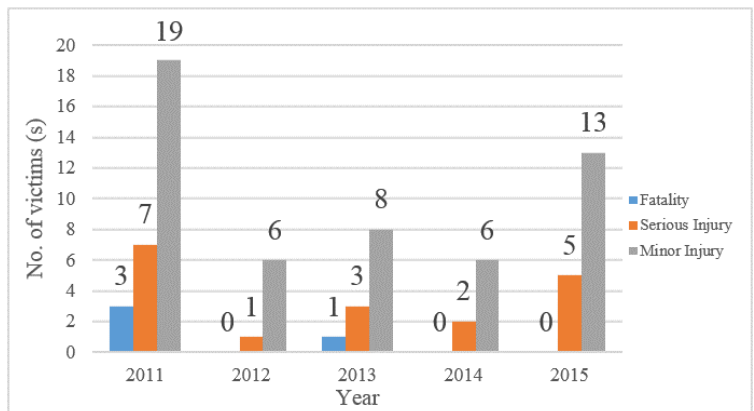

Figure 1. Jalan Gadong's RTA casualty trend from 2011 to 2015.

\section{Identification and ranking of RTA hotspots}

A GIS analysis method was developed to identify and rank RTA hotspots along Jalan Gadong. In itially, RTA data for Jalan Gadong was exported into ESRI ArcGIS 10.2 as a point shape file. A "Select by location" function in ArcGIS filtered out RTA points that were located outside of Jalan Gadong. For each RTA point, a proximity analysis was conducted by creating a buffer zone of a fixed radius centred at the RTA coordinates.

Previous studies have used arbitrary fixed distances to segment the road network for spatial analysis, irrespective of traffic speeds. However, the radius of the buffer zones in this study was taken to be equal to the Stopping Sight Distance (SSD) on Jalan Gadong. SSD is typically defined as the distance traversed by a vehicle from the time its driver perceives a hazard in the vehicle's path to the time the vehicle brakes to a stop [15]. It should be noted that the SSD is a proxy measure as vehicles do necessarily stop after that distance postcollision. However, noting that a large proportion of RTAs in Brunei are single-vehicle accidents [8], SSD has the advantage of reflecting the effect of speeds on the final resting locations of the vehicles, as the latter are more likely to be accurately recorded compared to say, the location of loss of control. To compute the SSD, the speed was chosen as the posted speed limit for the majority of Jalan Gadong $(65 \mathrm{~km} / \mathrm{hr})$, as no data on operating speeds was available. The perception-reaction time was taken as 1.5 seconds, while the longitudinal friction coefficient between vehicle tyres and road surface was taken as 0.33 [15], yielding an SSD value of $80 \mathrm{~m}$.

Closeness and buffering of RTA points resulted in many overlapping circles which was not helpful in identifying RTA hotspots. Hence, every group of overlapping circles were merged into a hotspot zone, as RTA locations within SSD distance of each other could reflect a particular hazardous zone. This process resulted in 12 RTA hotspot zones as shown in Figure 2. 


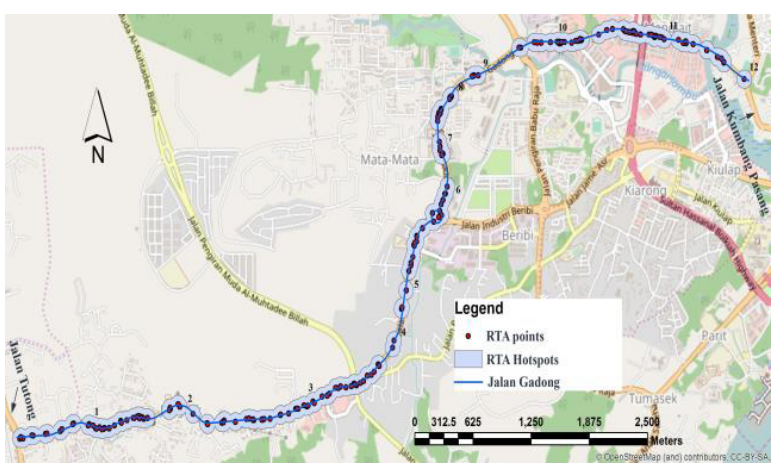

Figure 2. RTA hotspots along Jalan Gadong.

\section{Prioritisation of RTA hotspots}

With the help of a "Join" function in ArcGIS, RTA points located within each RTA hotspot zones, as shown in Figure 2, were automatically combined and counted. Moreover, the same jo in function helped to sum up the number of fatalities, serious injuries, minor injuries and no injuries related to RTA points located within each zone. For this research, four ranking schemes were used to prioritise RTA hotspots. The first ranking scheme was based on frequency of RTA points inside each hotspot zone. Equation (1) was developed for this scheme which involves the density of RTA within each zone and the number of years of data. It also involves SSD to account for the operating speed, longitudinal friction coefficient and gradient of the road, as well as the driver's perception-reaction time. These four parameters contribute significantly to the risk of having a traffic accident on the road.

$$
F=R T A \times(S S D / L) \times(1 / N)
$$

where:

$F=$ average number of yearly RTA within each hotspot zone per SSD,

$R T A=$ RTA count within the hotspot zone,

$S S D=$ Stopping Sight Distance,

$L=$ length of hotspot zone along the road centreline, and $N=$ number of years of data.

The second ranking scheme was developed based on the severity with in each RTA zone, which was adopted from an approach developed by Ref. [16]. Equation (2) was used to compute the risk factor within each zone.

$$
S=X+3 Y+5 Z
$$

where:

$S=$ severity

$X=$ total number of minor injury within each RTA hotspot zone,

$Y=$ total number of major injury within each RTA hotspot zone, and

$Z=$ total number of fatalities within each RTA hotspot zone.

The normalised severity (NS) is calculated from $S$ using Equation (3).

$$
N S=S \times(S S D / L) \times(1 / N)
$$

where:

$N S=$ normalised severity (severity per SSD length per year),

$S=$ severity,

$S S D=$ Stopping Sight Distance,

$L=$ length of hotspot zone along the road centreline, and $N=$ number of years of data.

The third ranking scheme was based on the socioeconomic impact of RTA within each hotspot zone. A formula, developed by Ref. [17], was adopted for the estimation of the socio-economic impact in BND as follows:

$$
S E I=(\text { Fatality } \times \$ 1,419,639)+
$$

$($ Serious injury $\times \$ 70,205)+($ Slight injury $\times \$ 9,119)+$

(No injury $\times \$ 3,300$ )

where:

$S E I=$ socio-economic impact.

The normalised socio-economic impact (NSEI) is calculated from SEI using Equation (5).

$$
N S E I=S E I \times(S S D / L) \times(1 / N)
$$

where:

NSEI = normalised socio-economic impact (Socioeconomic impact per SSD length per year),

$S E I=$ socio-economic impact,

$S S D=$ Stopping Sight Distance,

$L=$ length of hotspot zone along the road centreline, and $N=$ number of years of data.

The RTA hotspot zones were split into four hierarchical classes with equal number of zones. A colour scheme was then applied to all hotspot zones. This was to display visually the hierarchy of frequency, normalised severity and normalised socio-economic impact of RTA within each zone, as shown in Figure $3 a, b$ and $c$, respectively.

It is noticeable that the outputs from the three ranking schemes demonstrated some resemblance with each other. The results of NSEI and NS based schemes were more similar to each other than to those of the F based scheme. The reason for the difference in the results between the NS and NSEI based schemes is due to the consideration of non-injury cases in the latter while lacking this in the former scheme. In addition, the relative weight of fatality is higher in the latter than that in the former scheme. Therefore, the fourth ranking scheme was developed by combining once the frequency scheme and the normalised severity scheme, and another time the frequency scheme and the normalised socio-economic impact scheme, into two composite ranking schemes.

The acquired values of $F, N S$ and NSEI for Jalan Gadong were classified into four equal-percentile levels, resulting in four hierarchical levels for $F, N S$ and NSEI. The two composite ranking schemes exhibit the risk factor of each RTA hotspot zone using two risk assessment matrices that employ the four hierarchical levels of $F, N S$ and NSEI as shown in Figure $4 \mathrm{a}$ and $\mathrm{b}$. The risk assessment matrix has been modified from [18] based on road safety audit practices in New Zealand, which emphasise on prioritising accidents with higher severity of injuries. Then a colour scheme was assigned 
to hotspot zones, in order to display the hierarchy of risk factors of RTA hotspots based on NS and NSEI, as shown in Figures 5 and 6 respectively.

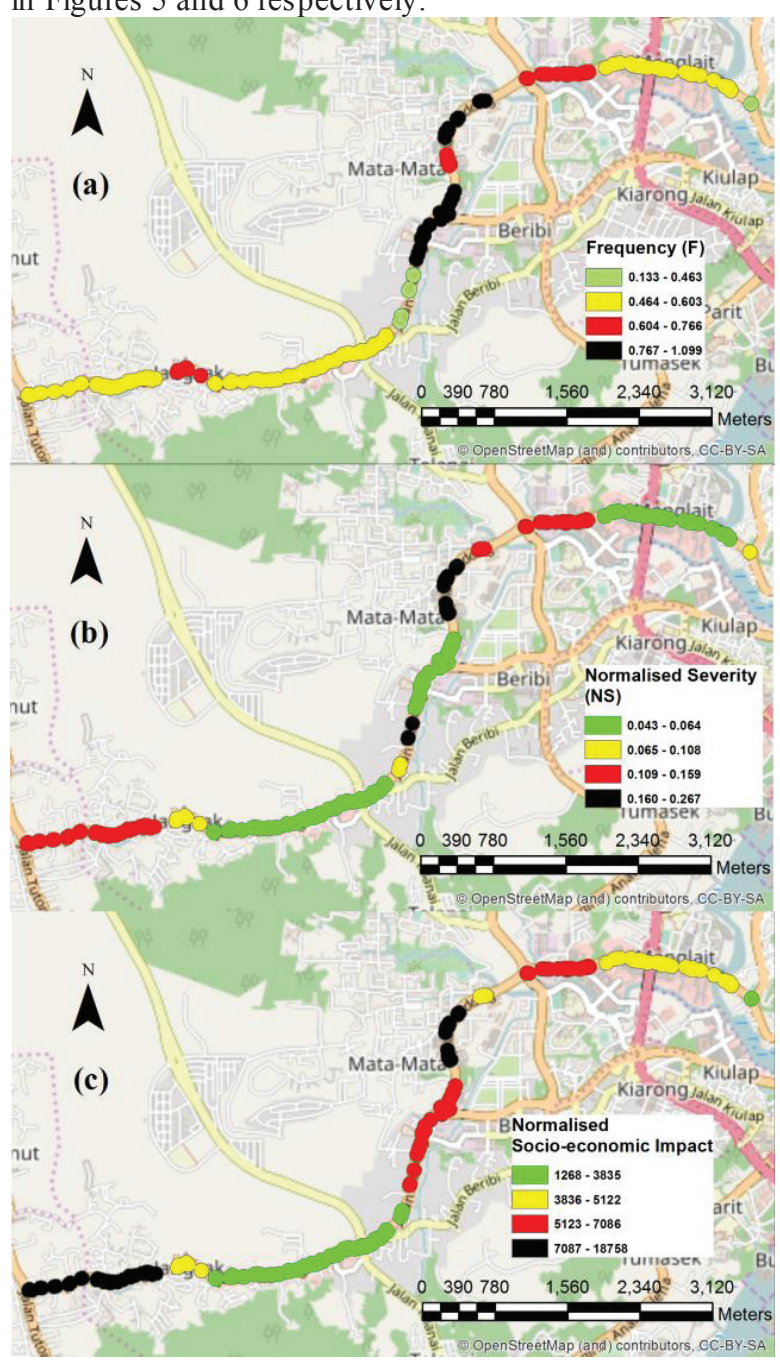

Figure 3. Prioritisation ( $a, b$ and $\mathrm{c}$ ) of Jalan Gadong RTA hotspots.

Frequency (F)

(a) $F>0.77 \quad 0.6<F \leq 0.77 \quad 0.46<F \leq 0.6 \quad 0 \leq F \leq 0.46$

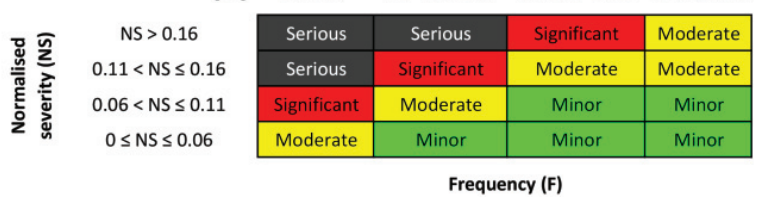

(b) $F>0.77 \quad 0.6<F \leq 0.77 \quad 0.46<F \leq 0.6 \quad 0 \leq F \leq 0.46$

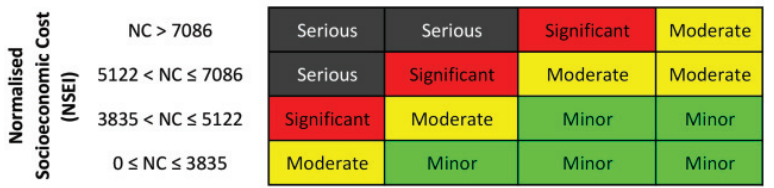

Figure 4. ( $a$ and $b$ ) Risk Assessment Matrices.

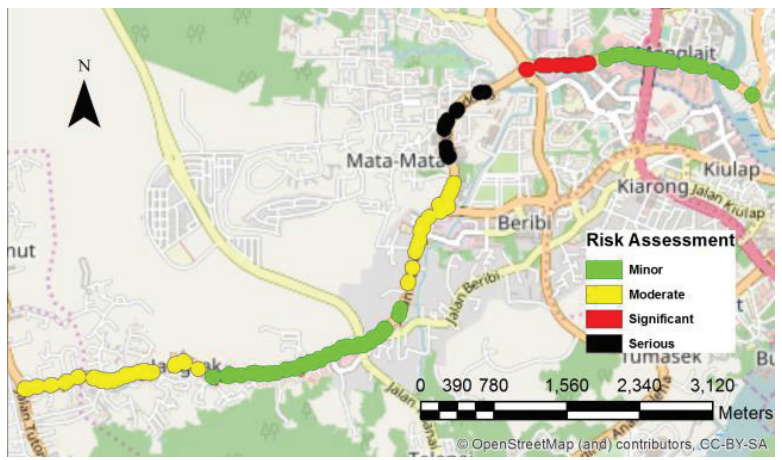

Figure 5. Normalised Severity-based Composite Ranking of Jalan Gadong RTA hotspots.

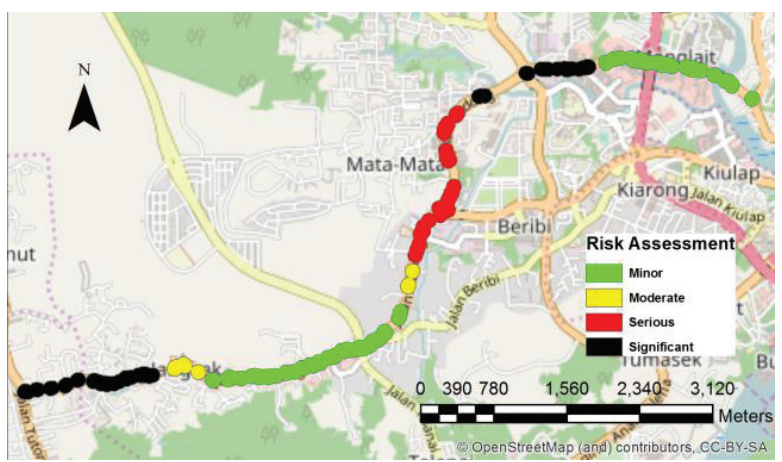

Figure 6. Normalised Socio-economic-based Composite

Ranking of Jalan Gadong RTA hotspots.

\section{Micro analysis of RTA hotspots}

As can be seen in Figure 2, the in itial stage of hotspot identification above resulted in a few continuous hotspot zones of up to 2 kilometres in length. A micro analysis method to achieve an improved visualisation of the spatial distribution of RTA within each identified hotspot zone was thus also developed. This method of data visualisation was used to determine road segments within the hotspot zone which had a relatively high density of RTA points. It could then facilitate subsequent road safety assessment and prioritisation of countermeasures, in light of limited resources for road safety imp rovement. The micro analysis method using GIS is described in the following.

The centreline of Jalan Gadong road was clipped by the RTA hotspot zones such that only the centreline portions within the RTA zones remained while those located outside the zones were eliminated. After that, the remaining centreline portions were split into equidistant segments, each of a length equal to the SSD. A "Near" tool in ArcGIS was used to project the RTA points onto each of the segments of Jalan Gadong. The "Join" function was then used to project RTA points onto the segments in all hotspot zones. To display the number of RTA points related to each segment, a symbology was assigned in the form of a column chart. A Python prelogic script code was applied to split the number of RTA points into 5 hierarchal classes. A colour scheme was used to show the hierarchy of these classes. In addition to the colour scheme, the column's length in the column chart was used to symbolise the count of RTA points 
related to each road-centreline segment, as shown in Figure 5.

Through this method, particular sections of Jalan Gadong with higher incidence of RTAs were identified. They were then brought forward for detailed road safety auditing and proposal of mitigation measures, with the aim of improving the safety of these sections of Jalan Gadong.

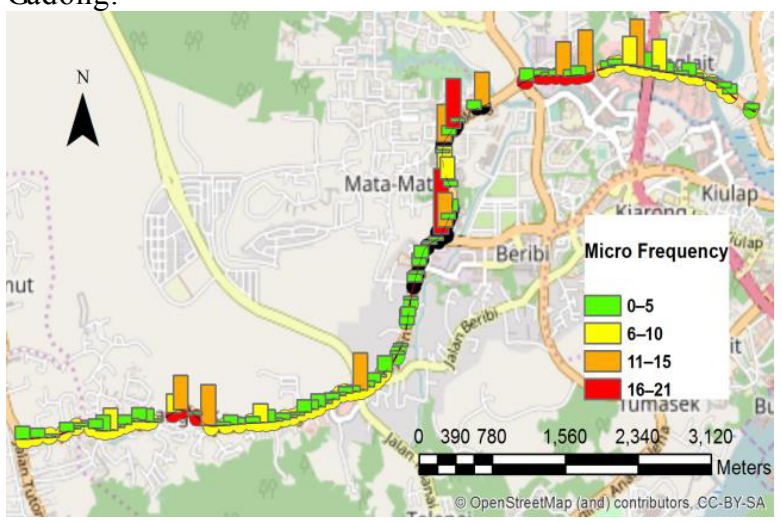

Figure 7. Micro Analysis of Jalan Jerudong RTA hotspots.

\section{Conclusions and recommendations}

Since Jalan Gadong is known to have a high number of accidents per kilo metre per year, the selection of this road as a case study for this research was helpful, especially when comparing the results of the three ranking schemes that are based on frequency, normalised severity and normalised socio-economic impact of RTA. The developed hotspot identification approach used SSD value as a radius to create buffer zone around each RTA points. This approach has a better justification than using arbitrary values of search radius and output cell size used in KDE method. However, merging overlapping buffer zones along Jalan Gadong created long RTA hotspots with a very high number of RTA points. This may impede the ability to prioritise the intervention and maintenance measures within the long stretch of the same RTA hotspot. The micro analysis method, explained in Section V and illustrated in Figure 7, was subsequently developed to ensure that the road segments with relatively high number of RTA could easily be identified for the purpose of road safety assessment and subsequent prioritisation.

Frequency, normalised severity and normalised socioeconomic impact of RTA within each zone were used to develop the three different ranking schemes in Figure $3 \mathrm{a}, \mathrm{b}$ and $\mathrm{c}$. The division by the length of the hotspot zone in (1), (3) and (5) was found helpful to prioritise RTA hotspots according to the density of RTA points, severity and socio-economic impact within each hotspot. For the same number of RTA points, severity and socio-economic impact per hotspot, the longer the hotspot zone, the less the density of RTA points within the zone, and the less the value of F, NS and NSEI in (1), (3) and (5), indicating less priority. The division by both the length of the hotspot zone and the number of years in (1), (3) and (5) may allow the prioritisation of RTA hotspots across many roads with different lengths of hotspot zones and different number of years of data. More research is recommended to calibrate (2) and (3) to the local conditions of Brunei Darussalam.

Due to the similarity between the results of the three ranking schemes based on frequency, normalised severity and normalised socioeconomic impact of RTA, two composite ranking schemes were developed to combine both the frequency and normalised severity, and the frequency and normalised socio-economic impact, of RTA within each identified hotspot into a composite risk factor. The composite ranking schemes can help priorit ise the development of intervention and maintenance programmes for road sections identified as RTA hotspots. Further research is needed to validate the hierarchy of risk factor identified at the two composite ranking schemes, and decide which one of them can provide a better prioritization of RTA hotspots.

It is anticipated that the methods and results developed in this study will facilitate a more efficient approach to improving the safety of the road network, by optimising the use of finite resources on priority hotspots to achieve more overall road safety gains.

\section{Acknowledgment}

The authors are grateful to the Brunei Research Council for their funding support and to the Brunei National Road Safety Council and the Royal Brunei Police Force for providing the necessary road safety data and information.

\section{References}

1. ICMR, Development of feasibility module for road traffic injuries surveillance. Indian Council of Medical Research Bulletin, 39, 41-50 (2009).

2. World Health Organization, Global status report on road safety 2015. (2015).

3. R. Ewing, S. Hamidi, and J.B. Grace, Urban sprawl as a risk factor in motor vehicle crashes. Urban Studies, (2014).

4. G. Yalcin and H.S. Duzgun, Spatial analys is of twowheeled vehicles traffic crashes: Osmaniye in Turkey. KSCE Journal of Civil Engineering, 19, 2225-2232 (2015).

5. Department of Statistics, Brunei Darussalam Statistical Yearbook. (2014).

6. Q. Bandial, Number of new vehicles increased by 19\% between 2011 and 2013, in The Brunei Times, (2015)

7. Centre for Strategic and Policy Studies, Review to Formulate a Roadmap and Draft National Masterplan for a Sustainable Land Transportation System for Brunei Darussalam. 5, (2014).

8. S.J. Tan, S. Ladi, E.M.M. Zahran, Y.H. Yap, and E.K.A. Rahman, A Review of Road Traffic Accident Statistics and Trends in Brunei Darussalam: presented at BICET 2016, Brunei Darussalam, (2016)

9. S. Ladi, C.D. Wijeyesekera, A.J. Brimico mbe, and Y. Li, Road traffic accident hotspot identification using modified Voronoi Process. Advances in 
Computing and Technology (AC\&T), 189-198 (2009).

10. G. Apparao, P. Mallikarjunareddy, and R.G. SSSV, Identification Of Accident Black Spots For National Highway Using GIS. International Journal of Scientific \& Technology Research, 2, 154-157 (2013).

11. R. Oulha, K. Brahimi, A. Boumediene, F. Dali, and M.A. Madouche, GIS Contribution to Identify Accident Black Spots on National Highway: Case Study of Wilaya of Mascara (Algeria). International Journal of Chemical, Environmental \& Biological Sciences (IJCEBS), 1, (2013).

12. S. Chainey, Examin ing the influence of cell size and bandwidth size on kernel density estimation crime hots pot maps for predicting spatial patterns of crime. Bulletin of the Geographical Society of Liege, 60 , 7-19 (2013).
13. L. Thakali, T.J. Kwon, and L. Fu, Identification of crash hotspots using kernel density estimation and kriging methods: a comparison. Journal of Modern Transportation, 23, 93-106 (2015).

14. Centre for Strategic and Policy Studies, Review to Formulate a Roadmap and Draft National Masterplan for a Sustainable Land Transportation System for Brunei Darussalam. 3, 31 \& 35 (2012).

15. C.A. O'Flaherty, Highways: Volume 1 Traffic Planning and Engineering. 3 ed, 369-370, (1996)

16. K. Geurts and G. Wets, Black Spot Analys is Methods:Literature Review. (2003).

17. Asian Development Bank, The Cost of Road Traffic Accidents in Brunei Darussalam. (2005).

18. NZ Transport Agency, Road safety audit procedures for projects. Interim release, (2013). 